\title{
MRI of Lower Limb Muscle in Patients with 4q35- linked Facio-Scapulo-Limb, Type 2 (the Same as a Facioscapuloperoneal or a FSHD1) Autosomal Dominant Muscular Dystrophy
}

\author{
Valery Kazakov ${ }^{1,2^{*}}$, Dmitry Rudenko ${ }^{1,2}$, Alexander Pozdnyakov ${ }^{3}$, Vladislav Kolynin ${ }^{1,2}$ and Tima Stuchevskaya ${ }^{1,2}$ \\ ${ }^{1}$ Department of Neurology, Pavlov State Medical University, St. Petersburg, Russia \\ ${ }^{2}$ Second Department of Neurology and Third Department of Neurology, City Hospital 2, St. Petersburg, Russia \\ ${ }^{3}$ Institute of Radiology, St. Petersburg, Russia
}

Correspondence to:

Valery Kazakov, $\mathrm{MD}, \mathrm{PhD}$

Professor of Clinical Neurology

Department of Neurology

Pavlov State Medical University

L. Tolstoy Str. 6/8, 197022 St. Petersburg, Russia

Tel(Office): +7 8125109381

Tel(Home): +7 8123232121

E-mail: valerykazakov@mail.ru

Received: June 21, 2016

Accepted: August 24, 2016

Published: August 26, 2016

Citation: Kazakov V, Rudenko D, Pozdnyakov A, Kolynin V, Stuchevskaya T. 2016. MRI of Lower Limb Muscle in Patients with 4q35linked Facio-Scapulo-Limb, Type 2 (the Same as a Facioscapuloperoneal or a FSHD1) Autosomal Dominant Muscular Dystrophy. J Neurol Exp Neurosci 2(2): 21-27.

Copyright: (C) 2016 Kazakov et al. This is an Open Access article distributed under the terms of the Creative Commons Attribution 4.0 International License (CC-BY) (http://creativecommons. org/licenses/by/4.0/) which permits commercial use, including reproduction, adaptation, and distribution of the article provided the original author and source are credited.

Published by United Scientific Group

\begin{abstract}
The aim of this study is to describe and correlate the clinical and MRI data in facio-scapulo-limb, type 2 (FSLD2) (the same as a facioscapuloperoneal or a FSHD1) family members. We studied 17 FSLD2 patients (10 men and 7 women, 17-73 years old; mean age $40 \pm 3.7)$ from 12 families with $4 \mathrm{q} 35$ p13E11 EcoRI/ BlnI deletion 13-28 kb. Five patients were presymptomatic $(\operatorname{Pr})$ and 12 were symptomatic. MRI of 20 muscles bilaterally in observed patients was performed on a 1.5-tesla Siemens Magneton Vision system using axial T1 and T2 weighted images.
\end{abstract}

MRI study of Pr patients did not show any definite pathological changes of the lower limb muscles. In symptomatic patients more often and severe were involvement of anterior compartment of the lower leg muscles, posterior thigh muscles, rectus femoris and sometime later the adductors of thigh and gastrocnemius (medial head) muscles with relative sparing especially of quadriceps (vastus lateralis, medialis and intermedius) and peroneus longus muscles and a lesser degree of gracilis, sartorius, gastrocnemius (lateral head) and soleus muscles and with complete sparing of the deep posterior compartment of the lower leg muscles. MRI may disclose early selective involvement of some posterior thigh and lower leg muscles that could not be detected by manual muscle strength testing in the patients with clinical pure facioscapuloperoneal phenotype. MRI findings there were not correlated ( $p>0.05$ ) with DNA fragment size, age, disease severity and disease duration, daily life work disability and myogenic phenotype. It is quite probably that the FSLD2 is a special type of muscular dystrophy.

\section{Keywords}

Facioscapuloperoneal muscular dystrophy, Muscle MRI, Selective muscle involvement

\section{Introduction}

In the recent years, several studies have reported the value of MRI in identifying patterns of muscle involvement in different types of muscular dystrophy [1-10] including the FSHD one [11-14]. In opinion of these authors [11-14] the muscle MRI examination is a powerful tool in the evaluation FSHD patients and may be helpful in clinical trials for the FSHD.

The aim of this study is to describe and correlate the clinical and MRI data in different FSLD2 families' members at different stages of the disorder. 


\section{Patients and Methods}

We studied 17 FSLD2 patients (10 men and 7 women, 17-73 years old, mean age $40 \pm 3.7$ ) from 12 families with 4q35 DNA fragment size (DFS) ranged from 13 to $28 \mathrm{~kb}$ (double digestion) except one presymptomatic (Pr) and two symptomatic patients (see below).

MRI of 20 muscles bilaterally in each patient was performed on a 1.5 - tesla Siemens Magnetom Vision system, using T1 weighted (TR $=832 \mathrm{~ms}, \mathrm{TE}=12 \mathrm{~ms}$, matrix $256 \mathrm{x}$ 192) and T2 weighted $(\mathrm{TR}=5312 \mathrm{~ms}, \mathrm{TE}=99 \mathrm{~ms}$, matrix 256 x 192) images. Axial $8 \mathrm{~mm}$ thick section and the $9 \mathrm{~mm}$ gap between slices of the thigh and lower leg muscles were created. Scanning time was within $30 \mathrm{~min}$ for each patient. 20 sections of muscle were analyzed with an emphasis on the mid-sections of the thighs and lower legs. For description of the muscle involvement the T1W images were used. The signal intensity was classified according to Jungbluth et al. [2, 3]: 0 = normal, 1 = mild with only traces of increased signal intensity, 2 = moderate with increased signal in less than 50\% of affected muscle, 3 = severe with increased signal intensity in more than $50 \%$ of affected muscle and $4=$ entire muscle replaced by abnormal signal. Control included 8 men $(5$ men and 3 women, aged 19-57; mean age $31 \pm 4.7$ years) without signs of neuromuscular diseases.

The next muscles were examined

Thigh: rectus femoris, vastus lateralis, intermedius and vastus medialis, gracilis, sartorius, semimembranosus, semitendinosus, biceps femoris and adductor muscles; Lower leg: tibialis anterior, extensor digitorum longus, extensor hallucis longus, peroneus longus, gastrocnemius, soleus, tibialis posterior, flexor digitorum longus, flexor hallucis longus. Normal cross-section anatomy at the level of the midthigh and the mid-calf was established according [15]. The correlations between the MRI (mean MRI severity score) and DNA fragment size (DFS), patient's age, muscle strength, disease duration, disease severity, myogenic phenotype and daily-life work disability (DLWD) were analysed by a nonparametric Spearman rank analysis. Muscle strength was measured manually according to Daniels et al. [16] using the MRC grading scale.

\section{Results}

Presymptomatic $(\mathrm{Pr})$ patients with facioscapular $(\mathrm{FS})(\mathrm{n}=1)$ and facioscapuloperoneal (FSP) phenotypes $(n=4)$

Clinical study

We studied 5 Pr patients; 3 men (Bab.- aged 17, DFS 26 kb; Lev.- aged 23, not DFS; and Sav.- aged 26, DFS 14 $\mathrm{kb}$ ) and 1 female (Kos.- aged 18, DFS $13 \mathrm{~kb}$ ) had a FSP phenotype and one man (Kuk.- aged 20, DFS $27 \mathrm{~kb}$ ) had a FS one. These patients did not complain of motor disturbances (even in response to the special test questionnaires used). On examination in all patients the minimal weakness and/ or atrophy of orbicularis oris muscle on the right or left side and slight atrophy of the lower part of trapezius and/ or serratus anterior muscles were revealed. In 4 patients very slight weakness of the anterior tibial muscles was found. These patients could not stand up fully on the heels and/or on the one heel. In all patients the deep tendon reflexes is excluded Axilles ones were decreased.

\section{MRI study}

MRI study of $\operatorname{Pr}$ patients using T1-weighted (T1W) and T2-weighted (T2W) images did not show any definite pathological changes of the lower limb muscles. However, in four Pr patients (Kos., Kuk., Lev. and Sav.) a mild increased signal intensity on $\mathrm{T} 2 \mathrm{~W}$ in some slices (from 10 to 17 ) of tibialis anterior and extensor digitorum longus only on the right side was revealed as compared to the control (Figure 1).

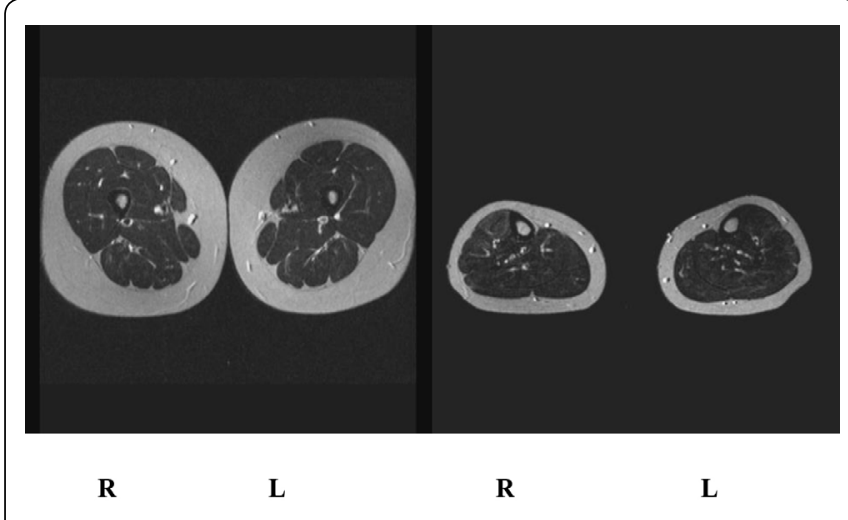

Figure 1: Axial T2 weighted images of thigh and lower leg muscles from Pr. patient Kos. The thighs are spared, lower leg showed increase signal intensity in tibialis anterior and extensor digitorum longus on the right side.

Note: R- right, L- left.

Symptomatic patients with facioscapuloperoneal (FSP) phenotype $(n=4)$

Clinical study

All patients (one-man S.- aged 73, DFS $24 \mathrm{~kb}$ and three women Ts. - aged 39, DFS 22 kb; Sh.- aged 42, DFS $24 \mathrm{~kb}$ (daughter of man S.) and K.- aged 41, DFS $20 \mathrm{~kb}$ had a pure FSP phenotype with severe weakness of the orbicularis oris muscle predominantly on the left or right side except the man S., who had only slight atrophy of the right half of upper lip. The patients could rise freely from the squatting position and stand up on the tip-toes, except the patient K., but could not stand up on the heels.

\section{MRI study}

Lower leg muscles: In all symptomatic patients with FSP phenotype the involvement of the anterior compartment of the lower leg muscles was found.

In patients K., S. and Sh. (Figure $2(\mathrm{~A}, \mathrm{C}, \mathrm{D})$ ) the symmetrical total involvement and in patient Ts. (Figure 2(B)) the asymmetrical moderate/severe involvement of the tibialis anterior and extensor digitorum longus muscles and in patients K. and S. (Figure 2(A, C)) a moderate/total affection of the extensor hallucis longus was found. Besides, in all these patients different degrees of affection of the gastrocnemius and soleus muscles was revealed. In patients Ts., S. and Sh. (Figure 2(BD)) a mild/moderate impairment of soleus on certain slices was found. In patient K. (Figure 2(A)) total fatty replacement 


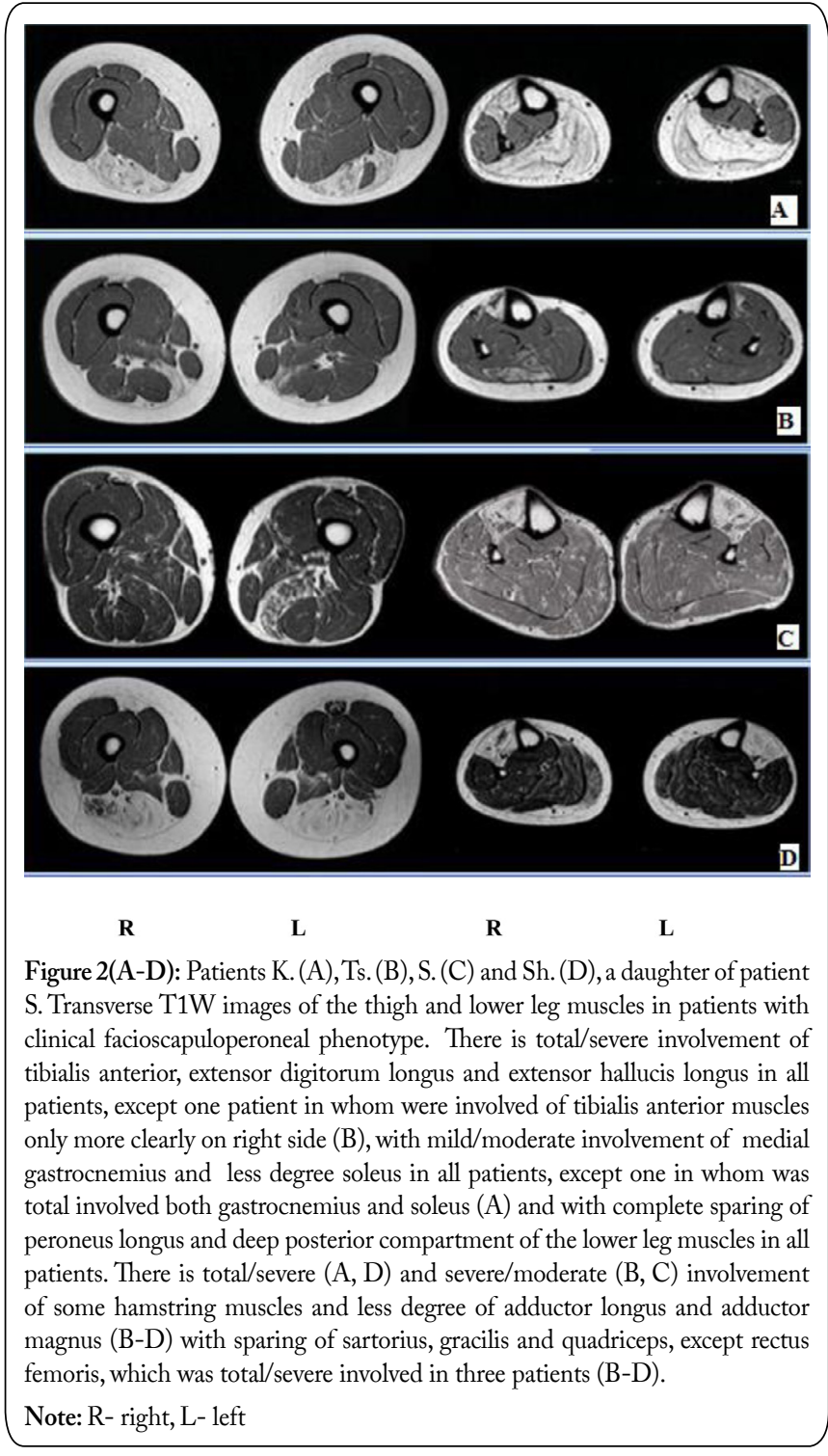

of this muscle as well as a total impairment of gastrocnemius (medial and lateral heads) muscles was observed. In patients $\mathrm{S}$. (Figure 2(B)) a mild/moderate partly involvement of medial head of gastrocnemius muscles was found; but in patient Sh. (Figure 2(D)), a daughter of S., a severe/moderate affection of gastrocnemius (medial heads) with sparing of the lateral heads of gastrocnemius muscles were revealed.

Thigh muscles: In all patients (Figure 2(A-D)) with clinical FSP phenotype different degrees of involvement of the posterior compartment thigh muscles were revealed.

In patient Sh. (Figure 2(D)) a bilateral total/severe affection of the semimembranosus, semitendinosus, a moderate/total involvement of biceps (long head), a moderate involvement of the adductor magnus on the left side and total affection of adductor longus muscles were found. Besides, the patient showed a total involvement of the rectus femoris muscle on the right side and moderate affection this muscle on the left side.

In patient S., aged 73 (Figure 2(C)), the father of patient Sh., the asymmetrical mild/moderate affection of the semimebranosus and a mild degree of involvement of the adductor magnus muscles were revealed more clearly on the left side. Besides, the patient showed total involvement of the rectus femoris more clearly on the right side.

In patient Ts. (Figure 2(B)) the bilateral total involvement of the semimebranosus and mild/moderate affection of adductor magnus were found. Besides, the patient showed a total involvement of the rectus femoris on the both sides as well as of adductor longus and on several slices of biceps femoris (long head) only on the one side.

In patient K. (Figure $2(\mathrm{~A})$ ) a bilateral total/severe involvement of the semimembranosus, semitendinosus and biceps femoris (long heads) and unilateral total affection of biceps femoris (short head) were found.

Patients with final facio-scapulo-peroneal-femoro (posterior thigh muscles)-gluteo (gluteus maximus) (FSPFG) $(\mathbf{n}=7)$ and facio-scapulo-peroneal-femoro (posterior thigh muscles)-gluteo (gluteus maximus) -humeral (FSPFGH) phenotypes $(\mathbf{n}=\mathbf{1})$

\section{Clinical study}

Three women (Ja. - aged 38, DFS 20 kb; Sav. - aged 62, DFS $14 \mathrm{~kb}$ (mother of Pr. patient Sav.) and Zol. - aged 61, DFS $20 \mathrm{~kb}$ (mother of patients Ja and Z), and four men (S. aged 42, DFS $28 \mathrm{~kb}$; Bel. - aged 61, DFS $13 \mathrm{~kb}$ (father of Pr. patient Kos.); F. - aged 43 not DFS and L. - aged 44, not DFS (father of Pr. Patient Lev.) had a final FSPFG phenotype. Only one patient Z- aged 36, DFS $20 \mathrm{~kb}$ (son of patient Zol. and brother of patient Ja) had a final FSPFGH phenotype with severe affection of quadriceps and biceps brachii muscles although in patient's mother and patient's sister these muscles were preserved. In all these patients with final phenotypes during many years (average 10-12) the FSP phenotype existed in clinical picture and later the posterior group of thigh muscles (semitendinosus, semimembranosus, biceps femoris) and gluteus maximus muscles were involved. Gluteus medius and quadriceps as well as a biceps brachii muscle were preserved except the patient $Z$. Thus, in these 7 patients the FSP phenotype gradually transferred into final FSPFG and in one patient $Z$.in FSPFGH phenotypes. All patients could rise from squatting position, except the patient $Z$. stand up on the tip-toes, and patient Sav. could not stand up on heels.

\section{MRI study}

Lower leg muscles: In all patients (Figure 3(A-D)) the total/severe involvement of anterior compartment of lower leg muscles except extensor hallucis longus (in three patients these muscles were spared) and moderate/total (in two patients in mild degree) impairment of gastrocnemius muscles, more often the medial head, with complete sparing of deep posterior compartment of lower leg muscles were found. The peroneus longus muscles were preserved as well except the patients Ja. and Bel. (Figure 3(A, D)) whose relevant muscles were involved to a mild degree.

Thigh muscles: As a rule, there was severe/total diffuse impairment of posterior thigh muscles [(semimembranosus, semitendinosus and biceps femoris (both heads)], adductor magnus and adductor longus with sparing of sartorius, gracillis (except the patients S., Sav., F. and Bel.) (Figure 3(B-D)) and 
relative preservation of quadriceps. In four patients (Ja., F., L. and Zol.) (Figure 3(A)) a severe/total involvement of rectus femoris and partly a moderate/severe affection (in patients $\mathrm{L}$, $\mathrm{S}$ and Bel.) (Figure 3(B, D)) of vastus medialis or partly mild/ moderate affection of vastus intermedius (in patients $\mathrm{F}, \mathrm{L}, \mathrm{S}$, Bel.) (Figure 3(B-D)) on some slices were observed.

\section{Comment: Clinical Phenotypes and MRI}

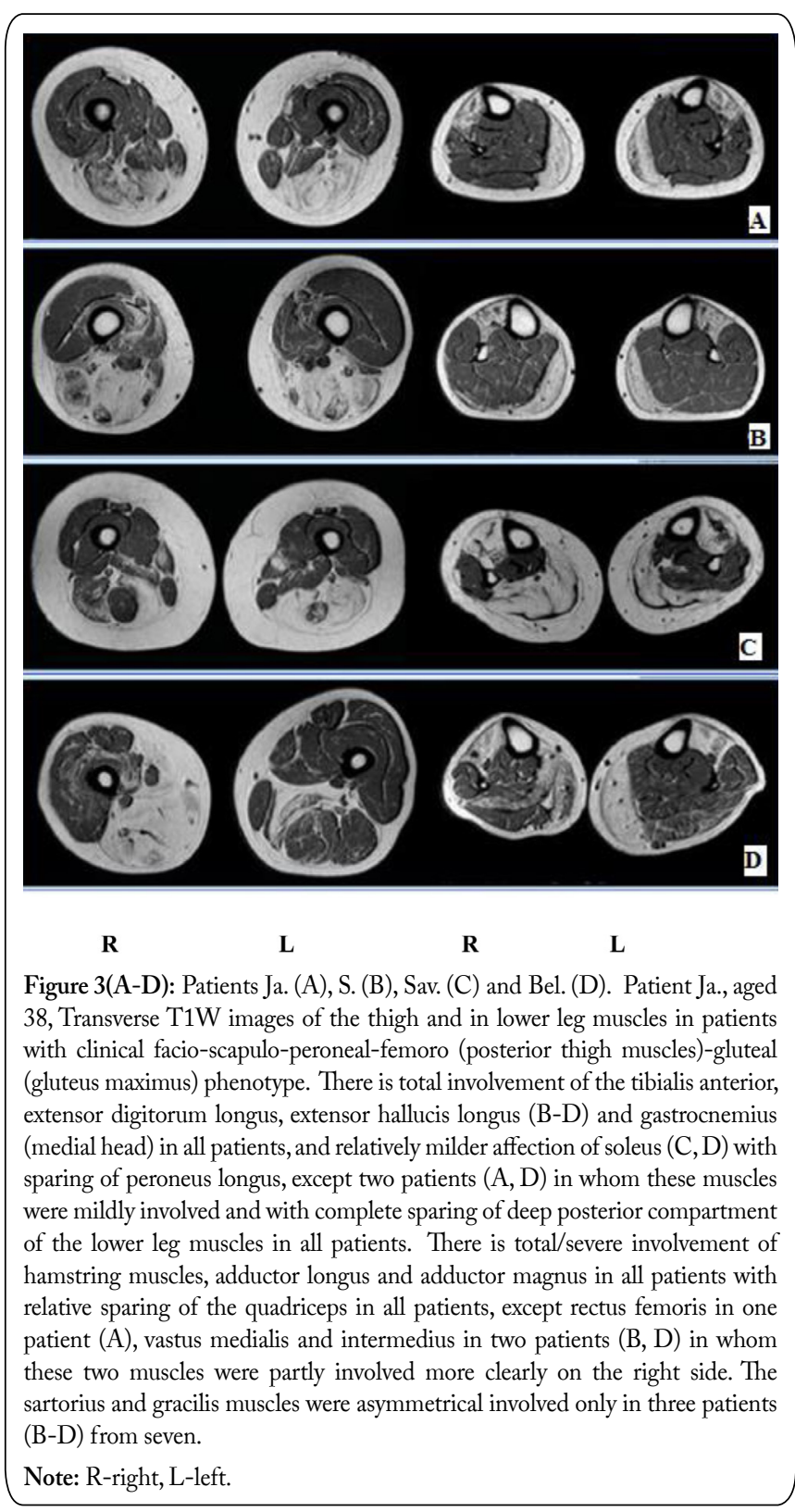

\section{Images}

In symptomatic patients the radiological muscle pattern involvement does not fully correlate with clinical pattern of muscle weakness (Table 1). In 11 from 12 patients the quadriceps muscles clinically had a clinically normal strength, although the total/severe of rectus femoris (in $83 \%$ on the both sides), vastus intermedius (in $50 \%$ on the right and $33 \%$ on the left), vastus medialis (in $42 \%$ on the right and $33 \%$ on the left) and vastus lateralis (in $25 \%$ on the both sides) were involved on MRI study with asymmetry affection for vastus intermedius and vastus medialis $17 \%$ and $9 \%$, respectively (Table 1 ). Clinically the hamstrings were involved in $70 \%$ patients. However, the total/severe affection of semimembranosus (in $100 \%$ on the both sides), biceps femoris caput longum (in $83 \%$ on the right and $75 \%$ on the left) and semitendinosus (in $67 \%$ on the right and $83 \%$ on the left) was revealed on MRI study with asymmetry affection for biceps femoris brevis and semitendinosus $17 \%$ and $16 \%$, respectively (Table 1 ). The tibialis anterior on the both sides and the extensor digitorum longus on the right side had total/ severe affection in all 12 patients (100\%) on clinical and MRI study. Clinically the soleus was affected in $16 \%$ patients and gastrocnemius was involved in $16 \%$ on the right and $25 \%$ on the left sides. However, m. soleus was slightly/moderately

Table 1: Frequency and asymmetry involvement of the lower limb muscles on clinical and MRI study in 12 symptomatic patients with FSLD2.

\begin{tabular}{|c|c|c|c|c|c|}
\hline \multicolumn{2}{|l|}{ Muscles } & $\begin{array}{c}\text { Total } \\
\text { clinical affected } \\
\text { muscles } \mathrm{N}(\%)\end{array}$ & $\begin{array}{c}\text { Total MRI } \\
\text { affected } \\
\text { muscles N (\%) }\end{array}$ & $\begin{array}{c}\text { Mean score } \\
\text { asymmetry } \\
\text { on clinical } \\
\text { study (\%) }\end{array}$ & $\begin{array}{c}\text { Mean score } \\
\text { asymmetry } \\
\text { on MRI } \\
\text { study }(\%)\end{array}$ \\
\hline \multirow{2}{*}{ Rect.fem. } & $d$ & $1(8 \%)$ & $10(83 \%)$ & \multirow{2}{*}{$0 \%$} & \multirow{2}{*}{$0 \%$} \\
\hline & $s$ & $1(8 \%)$ & $10(83 \%)$ & & \\
\hline \multirow{2}{*}{ Vastus int. } & $d$ & $1(8 \%)$ & $6(50 \%)$ & \multirow{2}{*}{$0 \%$} & \multirow{2}{*}{$17 \%$} \\
\hline & $s$ & $1(8 \%)$ & $4(33 \%)$ & & \\
\hline \multirow{2}{*}{ Vastus med. } & $d$ & $1(8 \%)$ & $5(42 \%)$ & \multirow{2}{*}{$0 \%$} & \multirow{2}{*}{$9 \%$} \\
\hline & $s$ & $1(8 \%)$ & $4(33 \%)$ & & \\
\hline \multirow{2}{*}{ Vastus lat. } & $d$ & $1(8 \%)$ & $3(25 \%)$ & \multirow{2}{*}{$0 \%$} & \multirow{2}{*}{$0 \%$} \\
\hline & $s$ & $1(8 \%)$ & $3(25 \%)$ & & \\
\hline \multirow{2}{*}{ Bic.fem. long. } & $d$ & $8(66 \%)$ & $10(83 \%)$ & \multirow{2}{*}{$9 \%$} & \multirow{2}{*}{$8 \%$} \\
\hline & $s$ & $9(75 \%)$ & $9(75 \%)$ & & \\
\hline \multirow{2}{*}{ Bic. fem. br. } & $d$ & $8(66 \%)$ & $7(58 \%)$ & \multirow{2}{*}{$9 \%$} & \multirow{2}{*}{$17 \%$} \\
\hline & $s$ & $9(75 \%)$ & $9(75 \%)$ & & \\
\hline \multirow{2}{*}{ Semimembr. } & $d$ & $8(66 \%)$ & $12(100 \%)$ & \multirow{2}{*}{$9 \%$} & \multirow{2}{*}{$0 \%$} \\
\hline & $s$ & $9(75 \%)$ & $12(100 \%)$ & & \\
\hline \multirow{2}{*}{ Semitend. } & $d$ & $8(66 \%)$ & $10(83 \%)$ & \multirow{2}{*}{$9 \%$} & \multirow{2}{*}{$16 \%$} \\
\hline & $s$ & $9(75 \%)$ & $8(67 \%)$ & & \\
\hline \multirow{2}{*}{ Tib.ant. } & $d$ & $12(100 \%)$ & $12(100 \%)$ & \multirow{2}{*}{$0 \%$} & \multirow{2}{*}{$0 \%$} \\
\hline & $s$ & $12(100 \%)$ & *10(100\%) & & \\
\hline \multirow{2}{*}{ Ext. dig. long. } & $d$ & $12(100 \%)$ & $12(100 \%)$ & \multirow{2}{*}{$0 \%$} & \multirow{2}{*}{$10 \%$} \\
\hline & $s$ & $12(100 \%)$ & $* 9(90 \%)$ & & \\
\hline \multirow{2}{*}{ Soleus } & $d$ & $2(16 \%)$ & $12(100 \%)$ & \multirow{2}{*}{$0 \%$} & 100 \\
\hline & $s$ & $2(16 \%)$ & $* 9(90 \%)$ & & $10 \%$ \\
\hline$C$ & $d$ & $2(16 \%)$ & $11(92 \%)$ & $00 \%$ & 20 \\
\hline Gustr. mea. & $s$ & $3(25 \%)$ & $* 9(90 \%)$ & & 270 \\
\hline An lat & $d$ & $2(16 \%)$ & $7(58 \%)$ & 00 & 20 \\
\hline Gastr.iat. & $s$ & $3(25 \%)$ & $* 6(60 \%)$ & $3 \%$ & $2 \%$ \\
\hline
\end{tabular}

Note: *- the indicated muscles were MRI examined only on the right side in 2 patients (from 12 ).

The muscle strength was uniform for all parts of quadriceps muscle (the rectus femoris and the vastus medialis, lateralis and intermedius muscles), for all knee flexor muscles (the biceps, semitendinosus, semimebranosus) and for medial and lateral gastrocnemius. d-right, s-left 
involved in $100 \%$ patients on the right and $90 \%$ on the left, and $\mathrm{m}$. gastrocnemius caput mediale had total/severe affection in $92 \%$ on the right and $90 \%$ on the left on MRI study (Table 1). Asymmetry affection for extensor digitorum longus and soleus was $10 \%$ (Table 1 ).

In patients with FSP phenotype the posterior thigh muscles clinically had a normal strength, although the total/ severe involvement of some hamstring muscles in all patients and less degree of adductor longus and magnus in 3 patients were revealed on MRI study (Figure 2(A-D)).

In 11 observed patients the quadriceps muscle showed a clinically normal strength, although the asymmetrical total/ severe (6 patients) or moderate ( 1 patient) involvement of rectus femoris and partly affection of vastus medialis or vastus intermedius (4 patients) was revealed on MRI study (Figure 2(A-D) and 3(A-D)).

In 3 patients with FSP and in 8 patients with FSPFG phenotypes the total/severe (8 patients) or moderate (1 patient) or mild ( 2 patients) involvement of gastrocnemius, more often the medial head, and to a lesser degree of the soleus muscles were revealed on MRI. However, clinically these muscles had a normal strength and all these patients, except for one with FSP and another patient with FSPFG phenotypes, could stand up on the toes.

The statistic a non-parametric Spearman rank analysis did not show significant correlation $(p>0.05)$ the severity of muscle involvement assessed by MRI with DFS ( $r=-0.19)$, patient's age $(r=-0.46)$, disease severity $(r=0.28)$ and disease duration $(r=-0.44)$, daily life work disability $(r=0.41)$ and myogenic phenotype $(r=0.55)$ (Table 1$)$. On the other hand, there was a reliable inverse correlation $(p<0.05)$ between muscle strength and MRI severity excluding the rectus femoris $(\mathrm{r}=-0.14 ; \mathrm{p}>0.05)$ and peroneus longus $(\mathrm{r}=-0.12 ; \mathrm{p}>0.05)$ muscles (Table 1).

\section{Discussion and Conclusion}

The muscle MRI of the FSLD2 patients showed more often and severe involvement of tibialis anterior, extensor digitorum longus, semimebranosus, long head of biceps femoris, semitendinosus, rectus femoris and sometime later the adductors of thigh and gastrocnemius (medial head) muscles with relative sparing especially of quadriceps and peroneus longus muscles in all patients and a lesser degree of gracilis, sartorius, gastrocnemius (lateral head) and soleus muscles and with complete sparing of the deep posterior compartment of the lower leg muscles (Table 1).

The MRI muscle pattern involvement does not fully correlate with clinical pattern of muscle weakness in symptomatic FSLD2 patients. MRI may disclose early selective involvement of some posterior thigh and lower leg muscles that could not be detected by manual muscle strength testing in the patients with clinical pure facioscapuloperoneal phenotype. MRI study confirmed our clinical data about the sequence involvement of the individual muscles in lower limbs in FSLD2 patients: early affection of the shin muscles (the muscles of the anterior compartment of the lower leg) and sometime later the hamstrings with sparing of the quadriceps $[17,18]$.

The principle MRI news are that some hamstrings muscles, especially the semimembranosus, biceps femoris longum, semitendinosus as well as rectus femoris, medial gastrocnemius and soleus are more severely and early affected even in patients having clinical pure facioscapuloperoneal phenotype. These data confirmed the opinion of some authors that "MR sensitivity is higher than clinical examination in detecting the involvement of individual muscles" [11] and "the MRI may disclose muscle involvement in FSHD that is not apparent on manual muscle testing" [12] and " $50 \%$ of movements assessed as normal on clinical examination associated with muscle abnormalities on MRI" [13].

Our data agree with MRI muscle study described [12] in 18 FSHD patients with DFS ranged from 12 to 31.5 $\mathrm{kb}$. MRI pattern of the lower limb muscles involvement in described patients is compatible with MRI muscle pattern in our symptomatic patients. Other authors [11] evaluated MR affection pelvic/lower limb muscles in 80 FSHD patients with DFS ranged $10-40 \mathrm{~kb}$. Although no specific patterns of muscle involvement were observed, the abdominal muscles, semimembranosus, tibialis anterior, biceps femoris (long head), semitendinosus and vastus medialis were more frequently and more severely affected while psoas major, iliacus, quadratus

Table 2: Correlation between the MRI severity and DNA fragment size (DFS), patient's age, myogenic phenotype, duration and severity disease, daily-life work disability (DLWD) and muscle strength in 10 symptomatic patients with FSLD2.

\begin{tabular}{|c|c|c|c|c|c|c|c|c|}
\hline & $\begin{array}{l}\frac{3}{10} \\
\text { s) } \\
\frac{1}{0}\end{array}$ & : & 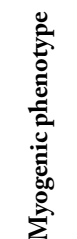 & 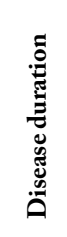 & 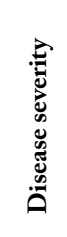 & $\frac{1}{a}$ & 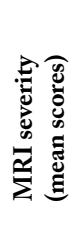 & 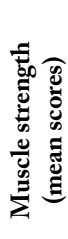 \\
\hline DFS kb & 1,00 & & & & & & & \\
\hline Patient's age & $-0,35$ & 1,00 & & & & & & \\
\hline $\begin{array}{l}\text { Myogenic } \\
\text { phenotype }\end{array}$ & $-0,08$ & $-0,19$ & 1,00 & & & & & \\
\hline $\begin{array}{l}\text { Duration } \\
\text { disease }\end{array}$ & $-0,35$ & $0,99^{*}$ & $-0,27$ & 1,00 & & & & \\
\hline $\begin{array}{l}\text { Severity } \\
\text { disease }\end{array}$ & 0,04 & 0,27 & 0,33 & 0,26 & 1,00 & & & \\
\hline DLWD & $-0,09$ & $-0,07$ & $0,80^{*}$ & $-0,13$ & $0,65^{*}$ & 1,00 & & \\
\hline $\begin{array}{l}\text { MRI severity } \\
\text { (mean scores) }\end{array}$ & $-0,19$ & $-0,46$ & 0,55 & $-0,44$ & 0,28 & 0,41 & 1,00 & \\
\hline $\begin{array}{l}\text { Muscle } \\
\text { strength } \\
\text { (mean MRC } \\
\text { scores) }\end{array}$ & 0,33 & 0,20 & $-0,78^{*}$ & 0,23 & $-0,46$ & $-0,59$ & $-0,86^{*}$ & 1,00 \\
\hline
\end{tabular}

Note: *- the correlation coefficients were significant $(p<0,05)$. The critical significance of the correlation coefficient R Spearman for $n=10$ is 0,632 . 
femoris, obturators, tibialis posterior, popliteus, iliopsoas, flexor digitorum longus and flexor hallucis longus were only rarely involved. On MRI at a field strength of 3T study of the lower limb muscles in 7 FSHD patients using T1W and T2W imaging the severe affection of the medial gastrocnemius, tibialis anterior and extensor digitorum longus was found [14]. However, the clinical myogenic phenotype was not described and MRI the thigh muscles were not analyzed.

In our study the MRI findings in muscle in 10 FSLD2 patients did not correlate with DFS, patient's age, severity and duration disease, daily life disability and myogenic phenotype but the significant correlate with manual muscle strength testing except of rectus femoris and peroneus longus (Table 2 and 3). On data [12] in 18 FSHD patients the MRI findings in muscles tended to correlate with disease duration, but did not correlate with DFS or age. There was a close correlation between muscle strength and MRI severity for different muscle groups (hip extension, abduction and adduction; knee flexion and extension; ankle dorsal flexion) except of muscles performing hip flexion and ankle plantar flexion. As well, the significant correlation between MRC scores and degree of MRI involvement of the tibialis anterior muscles was revealed in 7 FSHD patients by Kan et al. [14]. In last decade there are many articles in which was not found the significant correlation between DNA fragment size and phenotype, age of onset and

Table 3: Correlation between muscle strength and MRI severity involvement of the lower limb muscles in 12 symptomatic patients with FSLD2.

\begin{tabular}{|c|c|c|c|c|c|}
\hline \multirow{2}{*}{ Muscles } & \multicolumn{2}{|c|}{$\begin{array}{c}\text { Mean } \pm \text { SEM } \\
\text { MRC score }\end{array}$} & \multicolumn{2}{c|}{$\begin{array}{c}\text { Mean } \pm \text { SEM } \\
\text { MRI severity score }\end{array}$} & $\begin{array}{c}\text { Spearman } \\
\text { R }\end{array}$ \\
\cline { 2 - 5 } & D & S & D & S & \\
\hline Rectus femoris & $4.7 \pm 0.8$ & $4.7 \pm 0.8$ & $2.4 \pm 1.5$ & $2.4 \pm 1.5$ & -0.14 \\
\hline $\begin{array}{c}\text { Biceps femoris } \\
\text { longum }\end{array}$ & $3.4 \pm 1.5$ & $3.5 \pm 1.3$ & $2.2 \pm 1.5$ & $2.7 \pm 1.7$ & $-0.62^{*}$ \\
\hline $\begin{array}{c}\text { Biceps femoris } \\
\text { caput brevis }\end{array}$ & $3.4 \pm 1.5$ & $3.5 \pm 1.3$ & $1.9 \pm 1.9$ & $2.2 \pm 1.8$ & $-0.90^{*}$ \\
\hline Semimembranosus & $3.4 \pm 1.5$ & $3.5 \pm 1.3$ & $3.4 \pm 1.0$ & $3.4 \pm 0.9$ & $-0.49^{*}$ \\
\hline $\begin{array}{c}\text { Semitendinosus } \\
\text { Tibialis anterior } \\
\text { sinistra }\end{array}$ & $3.4 \pm 1.5$ & $3.5 \pm 1.3$ & $2.3 \pm 1.8$ & $2.4 \pm 1.5$ & $-0.65^{*}$ \\
\hline $\begin{array}{c}\text { Extensor digitorum } \\
\text { longus }\end{array}$ & $2.4 \pm 1,0$ & $1.4 \pm 1.2$ & $3.7 \pm 0.6$ & $3.1 \pm 1.4$ & $-0.53^{*}$ \\
\hline Peroneus longus & $4.4 \pm 0.8$ & $4.0 \pm 1.1$ & $0.4 \pm 0.6$ & $0.2 \pm 0.5$ & -0.12 \\
\hline Soleus & $4.7 \pm 0.7$ & $4.4 \pm 1.0$ & $1.9 \pm 1.1$ & $1.6 \pm 1.4$ & $-0.80^{*}$ \\
\hline $\begin{array}{c}\text { Gastrocnemius } \\
\text { mediale }\end{array}$ & $4.6 \pm 1.0$ & $4.3 \pm 1.2$ & $2.8 \pm 1.4$ & $2.7 \pm 1.5$ & $-0.63^{*}$ \\
\hline $\begin{array}{c}\text { Gastrocnemius } \\
\text { laterale }\end{array}$ & $4.6 \pm 1.0$ & $4.3 \pm 1.2$ & $1.5 \pm 1.7$ & $1.2 \pm 1.5$ & $-0.52^{*}$ \\
\hline
\end{tabular}

Note: * the correlation coefficients were significant $(p<0,05)$. The critical significance of the correlation coefficient R Spearman for $n=12$ is 0,497 .

$\mathrm{MRC}=$ Medical Research Council, MRI= magnetic resonance imaging. The muscle strength was uniform for all parts of quadriceps muscle (the rectus femoris and the vastus medialis, lateralis and intermedius muscles) and for all knee flexior muscles (the biceps, semitendinosus, semimebranosus) and for medial and lateral gastrocnemius. D-right, S-left the severity of the disease [19-25]. On Padberg's opinion “... since age of onset and severity of the disease roughly relates to the size of the deletion..."[26]. These data were confirmed by some geneticists in abstract called "Residual D4Z4 repeat size and D4Z4 methylation levels separate FSHD into two clinical severity classes" [27].

We saw the asymmetry involvement of some lower limbs muscle from $2 \%$ to $17 \%$ on MRI study and $9 \%$ on manual muscle testing (Table 1). In the opinion [12] asymmetrical involvement in FSHD patients occurs in $15 \%$ of the muscles on MRI and $6 \%$ on manual muscle testing and in the opinion [11] asymmetry of muscle affection in FSHD patients on MRI study is always evident in different muscle districts.

On MRI study of 4 Pr patients the slightest hyperintensity signal in TW2 imaging in tibialis anterior and extensor digitorum longus muscles only on the right side was found. Some authors found the hyperintensity signal in T2W imaging in clinically preserved posterior compartment of leg muscles in Pr patients and in symptomatic ones on the early stage with Miyoshi myopathy [9]. Authors connected this with myoedema. The same opinion was expressed by some other authors [24] who described increased signal intensity in both clinically preserved the semimembranosus muscles due to muscle edema in patient with muscular dystrophy. As well a hyperintensity signal due to muscular edema and/or inflammation was visible in two symptomatic patients with FSHD on the TIRM MR image in the soleus lateralis and soleus medialis muscles [14]. It is believed that the muscle MRI study in T2W imaging may find some abnormalities due to increased water in muscle (myoedema) in patients with different myopathies on the early stage and in $\operatorname{Pr}$ patients before clinical muscle weakness connected with fatty degeneration [9, 13, 28, 29].

Our clinical and MRI study shows that there are widespread involvements of the lower limb muscles in observed patients. In this connection, the inaccuracy of the term "facioscapuloperoneal" muscular dystrophy becomes evident. The name "Facio-scapulo-limb, type 2 muscular dystrophy (FSLD2), descending with a "jump", with initial facioscapuloperoneal phenotype" would be more correct [30]. FSLD2 is probably a clinical entity with specific clinical and MRI patterns of muscle involvement.

\section{References}

1. Birchall D, von der Hagen M, Bates D, Bushby K, Chinnery PF. 2005. Subclinical semitendinosus and obturators externus involvement defines an autosomal dominant myopathy with early respiratory failure. NeuromusculDisord 15(9-10):595-600.doi: 10.1016/j.nmd.2005.05.002

2. Jungbluth H, Sewry CA, Counsell S, Allsop J, Chattopadhyay A, et al. 2004. Magnetic resonance imaging of muscle in nemaline myopathy. Neuromuscul Disord 14(12): 779-784. doi: 10.1016/j.nmd.2004.08.005

3. Jungbluth H, Davis MR, Müller C, Counsell S, Allsop J, et al. 2004. Magnetic resonance imaging of muscle in congenital myopathies associated with RYR1 mutations. Neuromuscul Disord 14(12): 785-790. doi: 10.1016/j.nmd.2004.08.006

4. Jungbluth H, Zhou H, Sewry CA, Robb S, Treves S, et al. 2007. Centronuclear myopathy due to a de novo dominant mutation in the skeletal muscle ryanodine receptor (RYR1) gene. Neuromuscul Disord 17(4): 338-345. doi: 10.1016/j.nmd.2007.01.016 
5. Mercuri E, Talim B, Moghadaszadeh B, Petit N, Brockington M, et al. 2002. Clinical and imaging findings in six cases of congenital muscular dystrophy with rigid spine syndrome linked to chromosome $1 \mathrm{p}$ (RSMD1). Neuromuscul Disord 12(7-8): 631-638. doi: 10.1016/S09608966(02)00023-8

6. Mercuri E, Cini C, Pichiecchio A, Allsop J, Counsell S, et al. 2003. Muscle magnetic resonance imaging in patients with congenital muscular dystrophy and Ullrich phenotype. Neuromuscul Disord 13(78): 554-558. doi: 10.1016/S0960-8966(03)00091-9

7. Mercuri E, Lampe A, Allsop J, Knight R, Pane M, et al. 2005. Muscle MRI in Ullrich congenital muscular dystrophy and Bethlem myopathy. Neuromuscul Disord 15(4): 303-310. doi: 10.1016/j.nmd.2005.01.004

8. Mercuri E, Bushby K, Ricci E, Birchall D, Pane M, et al. 2005. Muscle MRI findings in patients with limb girdle muscular dystrophy with calpain 3 deficiency (LGMD2A) and early contractures. Neuromuscul Disord 15(2): 164-171. doi: 10.1016/j.nmd.2004.10.008

9. Brummer D, Walter MC, Palmbach M, Knirsch U, Karitzky J, et al. 2005. Long-term MRI and clinical follow-up of symptomatic and presymptomatic carriers of dysferlin gene mutations. Acta Myol 24(1): 6-16. doi:10.1007/s00415-005-0684-4

10. Schessl J, Medne L, Hu Y, Zou Y, Brown MJ, et al. 2007. MRI in DNM2-related centronuclear myopathy: Evidence for highly selective muscle involvement. Neuromuscul Disord 17(1): 28-32. doi: 10.1016/j. nmd.2006.09.013

11. Ricci E, Frusciante R, Mercuri E, Merico B, Di Giacopo R, et al. 2005. Lower limb involvement in FSHD: a MR study. Neuromuscul Disord 15(9-10): 709. doi: 10.1016/j.nmd.2005.06.006

12. Olsen DB, Gideon P, Jeppersen TD, Vissing J. 2006. Leg muscle involvement in facioscapulohumeral muscular dystrophy assessed by MRI. JNeurol 253(11): 1437-1441. doi: 10.1007/s00415-006-0230-z

13. Sookhoo S, Mackinnon I, Bushby K, Chinnery PF, Birchall D. 2007. MRI for the demonstration of subclinical muscle involvement in muscular dystrophy. Clin Radiol 62(2): 160-165. doi: 10.1016/j. crad.2006.08.012

14. Kan HE, Scheenen TW, Wohlgemuth M, Klomp DW, van LoosbroekWagenmans I, et al. 2009. Quantitative MR imaging in individual muscle involvement in facioscapulohumeral muscular dystrophy. Neuromuscul Disord 19(5): 357-362. doi: 10.1016/j.nmd.2009.02.009

15. Bergman RA, Afifi AK, Jew JJ, Reimann PC. 2014. Atlas of Human Anatomy in Cross Sections: Section 7- Lower Limb. In: D'Alessandro MP, Bergman RA (eds) Anatomy Atlases.

16. Daniels L, Williams M, Worthingham C. 1956. Muscle Testing. Techniques of Manual Examination (2 ${ }^{\text {nd }}$ ed.) Saunders, Elsevier, USA.

17. Kazakov VM. 1971. Facioscapulohumeral muscular dystrophy. Clinic and genetics. Thesis. Leningrad Pavlov Medical Institute, Russia, pp 234.

18. Kazakov V, Rudenko D, Katsev E, Sugita H, Goto K, et al. 2000. Facioscapulolimb muscular dystrophy (facioscapuloperoneal form of
FSHD) in Russian families. Phenotype/genotype correlation. Acta Myol 19: 69-79.

19. Goto K, Nishino I, Hayashi YK. 2004. Very low penetrance in 85 Japanese families with facioscapulohumeral muscular dystrophy $1 \mathrm{~A} . J$ Med Genet 41(1): e12. doi: 10.1136/jmg.2003.008755

20. Verschuere A, Laforet P, Pouget J. 2001. Facioscapulohumeral muscular dystrophy: correlation between clinical severity and genotype. Neuromuscul Disord 11: 634.

21. Busse K, Köhler J, Stegmann K, Pongratz D, Koch MC, et al. 2000. An inherited $4 \mathrm{q} 35-$-EcoRI-DNA fragment of $35 \mathrm{~kb}$ in a family with a sporadic case of facioscapulohumeral muscular dystrophy (FSHD). Neuromuscul Disord 10(3): 178-181. doi: 10.1016/S09608966(99)00102-9

22. Tonini MM, Passos-Bueno MR, Cerqueira A, Pavanello R, Vainzof $\mathrm{M}$, et al. 2002. Facioscapulohumeral (FSHD1) and other forms of muscular dystrophy in the same family: is there more in muscular dystrophy than meets the eye? Neuromuscul Disord 12(6): 554-557. doi: 10.1016/S0960-8966(02)00014-7

23. Tonini MM, Passos-Bueno MR, Cerqueira A, Matioli SR, Pavanello $\mathrm{R}$, et al. 2004. Asymptomatic carriers and gender differences in facioscapulohumeral; muscular dystrophy (FSHD). Neuromuscul Disord 14(1): 33-38. doi: 10.1016/j.nmd.2003.07.001

24. Felice KJ, North WA, Moore SA, Mathews KD. 2000. FSH dystrophy 4q35-deletion in patients presenting with facilal- sparing scapular myopathy. Neurology 54(10): 1927-1931.

25. Felice KJ, Jones JM, Conway SR. 2005. Facioscapulohumeral dystrophy presenting as infantile facial diplegia and late-onset limb-girdle myopathy in members of the same family. Muscle Nerve 32(3): 368-372.

26. Padberg GW.2004. Facioscapulohumeral muscular dystrophy: a clinical experience. In: Upadhyaya M, Cooper DN (eds) Facioscapulohumeral Muscular Dystrophy (FSHD): Clinical Medicine and Molecular Cell Biology. Garland Science/Bios Scientific Publishers, Taylor and Francis Group, New York, USA, pp 40-53.

27. van Overveld PGM, Enthoven L, Ricci E, Felicetti L, Jeanpierre M, et al. 2005. Risidual D4Z4 repeat size and D4Z4 methylation levels separate FSHD into two clinical severity classes. Neuromuscul Disord 15(9-10): 716-717. doi: 10.1016/j.nmd.2005.06.006

28. Zuo CS, Villafuerte RA, Henry ME, Butman M, Dobbins RL, et al. 2006. Proton and sodium MRI assessment of fluid level in calf tissue. $J$ Magn Reson Imaging 24(1): 191-196.

29. Ozsarlak O, Schepens E, Parizel PM, Van Goethem JW, Vanhoenacker F, et al. 2001. Hereditary neuromuscular diseases. Eur J Radiol 40(3): 184-197. doi: 10.1016/S0720-048X(01)00399-0

30. Kazakov V. 1995. History of the recognition and description of the facioscapulohumeral muscular dystrophy and on the priorities of Duchenne, Erb, Landouzy and Dejerine. Acta Cardiomiol 7: 79-84. 